\title{
Satellite Communications Supporting Internet of Remote Things
}

\author{
Mauro De Sanctis, Ernestina Cianca, Giuseppe Araniti, Igor Bisio, and Ramjee Prasad, Fellow, IEEE
}

\begin{abstract}
This paper focuses on the use of satellite communication systems for the support of Internet of Things (IoT). We refer to the IoT paradigm as the means to collect data from sensors or RFID and to send control messages to actuators. In many application scenarios, sensors and actuators are distributed over a very wide area; in some cases, they are located in remote areas where they are not served by terrestrial access networks and, as a consequence, the use of satellite communication systems becomes of paramount importance for the Internet of Remote Things (IoRT). The enabling factors of IoRT through satellite are: 1) the interoperability between satellite systems and sensors/actuators and 2) the support of IPv6 over satellite. Furthermore, radio resource management algorithms are required to enhance the efficiency of IoT over satellite. In this work, we provide an integrated view of satellite-based IoT, handling this topic as a jigsaw puzzle where the pieces to be assembled are represented by the following topics: MAC protocols for satellite routed sensor networks, efficient IPv6 support, heterogeneous networks interoperability, quality of service (QoS) management, and group-based communications.
\end{abstract}

Index Terms-Internet of Things (IoT), Internet of Remote Things (IoRT), machine-to-machine (M2M) communications, satellite communications, wireless sensor and actuator networks (WSANs).

\section{INTRODUCTION}

I T IS well recognized that one of the directions in the evolution of the Internet is toward the concept of Internet of Things (IoT) [1], [2]. The necessary condition to implement this new paradigm is the concept of smart objects. Smart objects are objects that have an "identity" in the network, which can be localized, which can process and communicate data and interact among themselves and the surrounding environment. This information processing and communication will support decision-making, improve situational awareness, increase operational efficiency of processes, and pave the way for new revolutionary applications in the most wide range of fields: industrial processes, logistics and infomobility, eco-sustainability, energy efficiency, remote assistance, and

Manuscript received February 18, 2015; revised April 02, 2015; accepted September 25, 2015. Date of publication October 05, 2015; date of current version January 20, 2016.

M. De Sanctis and E. Cianca are with the Department of Electronics Engineering, University of Roma "Tor Vergata," Rome 00175, Italy (e-mail: mauro.de.sanctis@uniroma2.it; ernestina.cianca@uniroma2.it).

G. Araniti is with the Department of Information Engineering, Infrastructure and Sustainable Energy (DIIES), University Mediterranea of Reggio Calabria, Reggio Calabria 89124, Italy (e-mail: araniti@unirc.it).

I. Bisio is with the Department of Telecommunication, Electronic, Electrical Engineering and Naval Architecture, University of Genoa, Genoa 16146, Italy (e-mail: igor.bisio@unige.it).

R. Prasad is with the Center for TeleInFrastruktur (CTIF), Aalborg University, Aalborg 9100, Denmark (e-mail: prasad@es.aau.dk).

Digital Object Identifier 10.1109/JIOT.2015.2487046
TABLE I

EXAMPLES OF SECTORS AND SERVICES IN IoT

\begin{tabular}{|c|c|c|c|}
\hline $\begin{array}{l}\text { Service } \\
\text { sector }\end{array}$ & $\begin{array}{l}\text { Application } \\
\text { group }\end{array}$ & Location & Devices \\
\hline Buildings & $\begin{array}{c}\text { Commercial, } \\
\text { institutional, } \\
\text { industrial }\end{array}$ & $\begin{array}{l}\text { Office, school, } \\
\text { hospital, } \\
\text { airport, } \\
\text { stadium }\end{array}$ & $\begin{array}{l}\text { Lighting, fire } \\
\text { \& safety } \\
\text { tools }\end{array}$ \\
\hline Energy & $\begin{array}{l}\text { Generation, } \\
\text { distribution, } \\
\text { demand }\end{array}$ & $\begin{array}{l}\text { Solar power } \\
\text { plant, wind } \\
\text { power plant } \\
\text { (inshore or } \\
\text { offshore), } \\
\text { power grid, } \\
\text { pipelines }\end{array}$ & $\begin{array}{c}\text { Turbines, } \\
\text { windmills, } \\
\text { batteries, } \\
\text { UPS, power } \\
\text { meters }\end{array}$ \\
\hline Healthcare & $\begin{array}{l}\text { Public care, } \\
\text { home care }\end{array}$ & $\begin{array}{l}\text { Hospitals, } \\
\text { home }\end{array}$ & $\begin{array}{l}\text { Electro- } \\
\text { medical } \\
\text { equipments }\end{array}$ \\
\hline Industrial & $\begin{array}{l}\text { Process } \\
\text { control, } \\
\text { process } \\
\text { automation }\end{array}$ & $\begin{array}{c}\text { Extractive site, } \\
\text { manufacturing } \\
\text { plant }\end{array}$ & $\begin{array}{l}\text { Pumps, } \\
\text { valves, } \\
\text { motors, } \\
\text { packaging }\end{array}$ \\
\hline $\begin{array}{c}\text { Transportati } \\
\text { on }\end{array}$ & $\begin{array}{c}\text { Terrestrial, } \\
\text { aeronautical, } \\
\text { maritime }\end{array}$ & $\begin{array}{l}\text { Ground, air, } \\
\text { sea, road, } \\
\text { railroad }\end{array}$ & $\begin{array}{l}\text { Cars, ships, } \\
\text { airplanes, } \\
\text { traffic lights, } \\
\text { toll booth }\end{array}$ \\
\hline Retail & $\begin{array}{c}\text { Stores, } \\
\text { specialty, } \\
\text { hospitality }\end{array}$ & $\begin{array}{c}\text { Cinema, } \\
\text { theatre, fuel } \\
\text { station, } \\
\text { supermarket, } \\
\text { shopping } \\
\text { center, hotel, } \\
\text { restaurant }\end{array}$ & $\begin{array}{l}\text { Vending } \\
\text { machines, } \\
\text { tags, POS } \\
\text { terminals, } \\
\text { cash registers }\end{array}$ \\
\hline $\begin{array}{l}\text { Public and } \\
\text { Safety } \\
\text { security }\end{array}$ & $\begin{array}{l}\text { Emergency } \\
\text { services, } \\
\text { law } \\
\text { enforcement, } \\
\text { monitoring }\end{array}$ & $\begin{array}{l}\text { Country level, } \\
\text { city level, } \\
\text { environment, } \\
\text { battlefield }\end{array}$ & $\begin{array}{c}\text { Jeep, car, } \\
\text { ambulance, } \\
\text { environment } \\
\text { sensor }\end{array}$ \\
\hline $\begin{array}{l}\text { ICT and } \\
\text { networks }\end{array}$ & $\begin{array}{c}\text { Public, } \\
\text { enterprise, } \\
\text { e- } \\
\text { commerce }\end{array}$ & $\begin{array}{c}\text { Data center, } \\
\text { service } \\
\text { providers, } \\
\text { telecomm. } \\
\text { networks, } \\
\text { office } \\
\end{array}$ & $\begin{array}{l}\text { Servers, } \\
\text { routers, PC, } \\
\text { tablets, } \\
\text { phones }\end{array}$ \\
\hline
\end{tabular}

environmental monitoring. Sectors and services for IoT are listed in Table I. The capability of smart objects to communicate and interact each other without the human intervention, the so-called machine-to-machine (M2M) communication or machine-type communication (MTC), according to the definition provided by the Third-Generation Partnership Project (3GPP) [3], is a fundamental part of IoT. In this paper, both IoT and M2M acronyms will be used. In particular, we will use the term IoT when the focus is more on the general capability of Internet-connecting any kind of object in order to create an unprecedented access and exchange of information; we will use 

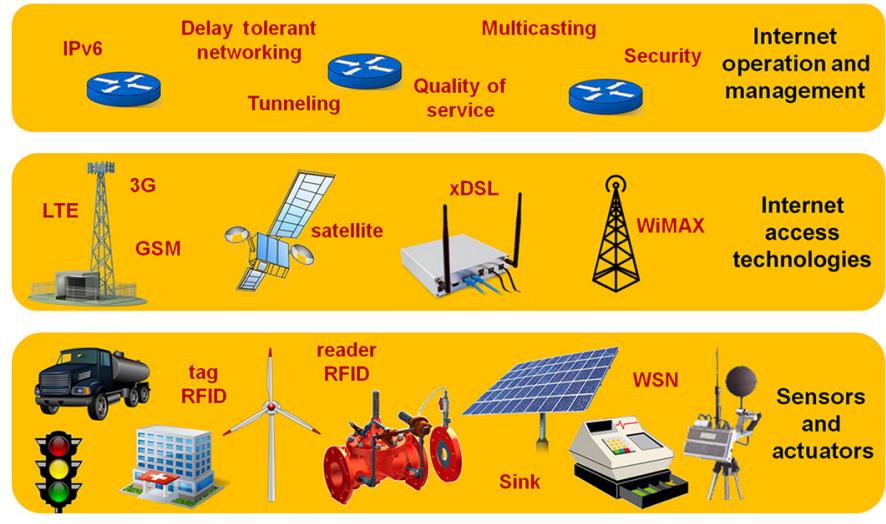

Fig. 1. System view of IoT.

the term M2M when the focus is on the more specific aspect of the IoT related to the communication of machines (i.e., a smart object) with each other or with a server on the other end of the communication link.

IoT and M2M applications have their own very unique features such as diverse service requirements, group-based communications, low or no mobility, time-controlled, time-tolerant, small data transmission, secure connection, monitoring, priority alarm messages, low energy consumption, and low cost [1]-[3]. These service requirements dictate the architectural design of the communication network, e.g., the selection of the Internet access technology. In particular, IoT is based on IPv6 and can exploit operation and management protocols for group-oriented communications, security, and quality of service (QoS) (see Fig. 1).

In this framework, satellite communications have the potential to play an important role for different reasons.

1) First of all, smart objects are often remote or they are dispersed over a wide geographical area or they are inaccessible (see possible locations in Table I). We refer to this particular situation of IoT as the Internet of (Remote) Things (IoRT). In IoRT, satellite communication provides a more cost-effective solution with respect to other terrestrial technologies to their interconnection and communication with the "the rest of the world" [4], [5].

2) As mentioned in [6] and [7], group-based communications is a transmission mode that will be important in several IoT and M2M applications where smart objects could be grouped according to the task and/or the information that have to receive. In such a case, network operators should optimize the volume of data to send when IoT/M2M devices need to receive the same message. This kind of applications can be naturally supported via satellite by exploiting broadcast (i.e., toward all nodes of the whole network), multicast (i.e., toward a portion of nodes of the whole network), or geocast (i.e., toward a portion of nodes placed in a given area of the network) transmissions.

3) In some applications, such as the smart grid, utilities need to implement the highest availability of data communications that is possible within reasonable cost. This requires redundant connections at critical sites. A terrestrial-only redundant approach is not sufficient, as severe disruptions on the ground can disable or destroy both fixed and wireless infrastructures alike, no matter the redundancy. Satellite could provide a true alternative path.

4) Existing IoT/M2M applications generally target low data rate transmission and, hence, current low bandwidth satellite infrastructures can be effectively reused.

As a matter of fact, IoT/M2M communication via satellite is a reality and represents a great opportunity for the satellite market. A comprehensive global report on the IoT/M2M via satellite services markets from global industry analysts states that, driven by the growing need of global businesses and governments to track, monitor, and exercise control over geographically dispersed fixed as well as mobile assets, the global market for satellite M2M services is projected to reach 1.7 billion dollars by 2017 [8]. Different satellite operators already provide integrated and complete M2M solution packages based on satellite [9].

However, new applications are emerging or the same applications are evolving toward scenarios that have different requirements (i.e., more stringent latency/reliability/data rate requirements) that are not met by the current implementations of M2M via satellite, which provide low data rate and access to few nodes via proprietary standards [10], [11]. For instance, in the current grid, substation and distribution automation is mostly performed through supervisory control and data acquisition (SCADA). With SCADA, a substation collects local measurements and delivers them to the application in the control centre, managed by human operators. Communication links between the individual substations and the control center form a star topology, and the collected data are stored in a centralized database. The low data rates on links (up to a few kilobits per second), and the latency up to 1-2 s does not allow to meet the stringent latency requirements (in the range $100 \mathrm{~ms}-1 \mathrm{~s}$ ) of most of the smart grid applications devoted to give insight into the grid dynamics through the monitoring of the energy load distribution across all substations and customer locations. Considering as real-time an application with a latency requirement $<100 \mathrm{~ms}$, we will refer as almost realtime an application with a latency requirement $<1 \mathrm{~s}$. Moreover, in several M2M, scenarios is emerging the need to manage more data-intensive applications such as possibility to send surveillance video almost in real-time from remote locations and provision to remote worker/asset management of additional communication capabilities, such as exchange of workflow information, emails, and electronic forms.

However, also in these more challenging scenarios, satellite communication has the potential to play a key role. Recent advances in satellite communication technology have dispelled some misconceptions on the use of satellite, related to the reliability and latency. Satellite networks are nowadays capable of providing 0.9999 availability. This is in part due to the use of adaptive coding and modulation (ACM) techniques as foreseen in the DVB-S2 standard [12], [13], which allows to guarantee outbound throughput even during inclement weather conditions. Moreover, a round trip time (RTT) of 600-700 ms through a geostationary earth orbit (GEO) satellite link is only few hundred milliseconds higher than the RTT of a long 
terrestrial link (100-200 ms), and it is still suitable for several M2M applications, including substation automation, distribution automation, and advanced metering infrastructure (AMI) [14], [15].

A recent feasibility study has shown that a low earth orbit (LEO) constellation of satellites could be effectively used for distributed control and automation in a smart grid scenario, meeting the stringent latency requirements. Moreover, the use of LEO constellations could help in meeting more stringent latency requirements in case of voice and video transmission [16], [17].

Nevertheless, to make an effective IoT via satellite, several issues are still open and need an answer.

This is the first paper, to the best the authors' knowledge, which makes the effort to put together the research works on satellite communication in the vision of IoT and M2M applications. In particular, the following issues are discussed: 1) the development of specialized MAC protocols for the access of sensor nodes to satellite resources; 2) the use of IPv6 over satellite; 3 ) interoperability between satellite and terrestrial networks; and 4) resource allocation and transmission management of group-based communications.

This paper is organized as follows. Section II describes some of the IoT scenarios where satellite could play a key role. Section III presents an overview of the research activity on specialized MAC protocols for the access of sensor nodes to satellite resources and the support of IPv6 over satellite. In Section IV, the more general issue of the interoperability of satellite and terrestrial networks is reviewed and some novel ideas are presented. Section V reports the main research results on group-based communications. Finally, in Section VI, a conclusion is drawn.

\section{IoRT SCEnARIos Where SATEllite Is A KEY ELEMENT}

There are many applications based on the IoT paradigm [18]. In this section, we select three typical applications of IoRT where the use of the satellite is of paramount importance: 1) smart grid; 2) environmental monitoring; and 3) emergency management. For each specific application, we present the current and/or the possible future role of the satellite. The objective is to highlight if current generation of IoRT via satellite can meet the specific application requirements and in case it cannot, to identify challenges and open issues toward an effective use of IoRT via satellite.

\section{A. Smart Grid}

One of the main efforts toward the implementation of a low-carbon society is the implementation of the smart grid concept [19]. The power grid becomes "smart" when it is able to react and adapt to any event occurring in any point of the grid (generation, transmission, distribution, and consumption) by adopting proper strategies and countermeasures. This capability is fundamental to the following.

1) Make the power production, transmission, distribution more energy efficient.
2) Fulfill the growing demand for energy with the same or higher reliability, still keeping low the operational costs. The Italian and US blackout in 2003 proved the limits of the traditional grid to provide reliable services.

3) Provide more transparent services to the user which must play an important role both as consumer and as producer; in fact, a new figure arises in the future power grid, i.e., the "prosumer", which is consumer and also producer of renewable energy.

To make the smart grid concept a reality, a bidirectional flow of information is needed among the different actors of the grid at different points of it. Many of the elements of a smart grid are already available, including smart meters, automated monitoring systems, and power management systems. The communication network has a crucial role and its cost and performance will greatly influence utilities' revenues and the capability of the new grid to meet its ambitious objectives. For sure, the communication network will be heterogeneous, including wired and wireless segments, and private and public solutions. In this framework, satellite could represent a viable and cost-effective solution in different scenarios, such as the following.

1) Structural and functional monitoring of offshore wind farms or solar energy systems in desert areas.

2) Back-up link when very high availability is required. Note that nowadays about $10 \%$ of electricity consumption requires $99.9999 \%$ of reliability and both numbers are expected to increase.

3) Remote monitoring and automated control of substations located in remote areas.

As a matter of fact, satellites are already used by utilities in the current grid and also by other types of companies involved in the deployment of renewable energy generation. In particular, M2M via satellite is mainly used today for: 1) providing back-up links, when high reliability is required and 2) remote monitoring and automated control of substations located in remote areas. In the current grid, remote monitoring and automated control of substations is implemented through the SCADA system, which includes several remote terminal units (RTUs) and intelligent electronic devices (IEDs), a master server, and a human-machine interface for the interaction with the human operator [20], [21]. The SCADA master server cyclically queries the operating and status data from the grid. It is usually a low data rate service, low duty cycle, and remote terminals can be geographically dispersed in rural areas. Therefore, as outlined in [22], a satellite-based solution can provide a cost-effective solution for providing a communication infrastructure to implement SCADA systems, especially in rural areas of the distribution network. Satellite connectivity has been used for years in SCADA applications for remote locations in oil and gas and other energy exploration areas. Traditional private satellite networks work well for these low data rate (for a basic SCADA service, a data rate of $128 \mathrm{~kb} / \mathrm{s}$ is enough), hard-to-reach application delivering secure and ubiquitous coverage inexpensively [23], [24]. The same hold for electric substation automation. It can act as both the primary communication link and a back-up link [25].

On the other hand, the current slow central network control based upon SCADA systems is no longer sufficient to 
TABLE II

REQuirements of SEVERAl Functionalities of the SMART GRID

\begin{tabular}{|l|l|l|c|}
\hline Functionality & Data rate $(\mathrm{kbps})$ & Latency & Reliability (\%) \\
\hline AMI & $\begin{array}{l}<100(500 \mathrm{kbps} \\
\text { for the backbone })\end{array}$ & $<15 \mathrm{~s}$ & $<99.99$ \\
\hline $\begin{array}{l}\text { Demand } \\
\text { response }\end{array}$ & $<100$ & $\begin{array}{l}\text { Several } \\
\text { minutes }\end{array}$ & $<99.99$ \\
\hline WASA & $<1500$ & $<200 \mathrm{~ms}$ & $<99.9999$ \\
\hline $\begin{array}{l}\text { Distributed } \\
\text { generators }\end{array}$ & $<56$ & $<15 \mathrm{~s}$ & $<99.99$ \\
\hline
\end{tabular}

implement the new concept of wide-area situational awareness (WASA), which is the use of several types of sensors along the grid to collect data about the state of the grid and by processing these data, being able to act effectively or predict future problems. Moreover, the WASA concept will be heavily based on the wide-area measurement systems (WAMSs) which have the task to collect synchronized measurements from sophisticated sensors, called phasor measurement unit (PMU) with the objective to have an almost real-time monitoring of the grid state [26]. In order to follow almost real-time the dynamics of the grid, measurements from the PMUs should be reported 2060 times per second. It is worth noting that traditional SCADA systems usually report the data with a frequency that ranges from seconds to minutes. Moreover, latency of the order of 1$2 \mathrm{~s}$ is usually accepted in traditional SCADA implementations. Table II shows the main requirements of several functionalities of the smart grid. The WASA concept is definitely more challenging in terms of reliability, latency, and also data rates and the current implementation of $\mathrm{M} 2 \mathrm{M}$ via satellite might be no longer suitable. Nevertheless, could satellite still play a role in this emerging smart grid scenario? What are the critical issues or key elements that should be included in a new generation of M2M via satellite systems to be effectively used in a smart grid scenario? Some studies have been performed to give an answer to the above-mentioned questions.

In [27], IP data services of Globalstar constellation (orbit altitude $1410 \mathrm{~km}$, data rate $7.2 \mathrm{~kb} / \mathrm{s}$ ) were assessed through an experimental test-bed using a prototype IED. The conclusion was that Globalstar can barely meet the stringent delay requirements for time critical applications in smart distribution networks due to its limited bandwidth and orbit altitude. A different conclusion was drawn in [16], where a LEO satellite-based network infrastructure is proposed to support a recently suggested active network management solutions, the autonomous regional active network management system (AuRA-NMS) control. However, the analysis in [16] was not limited to a specific existing LEO-based constellation, but they presented a general analytical model for the network delay and the loss behavior of a LEO network. In [16], several IP data services with diverse traffic characteristics and patterns over LEO networks have been evaluated for both regular and emergency operational scenarios under a range of delay and loss conditions. The performed feasibility study has shown that a LEO-based solution can meet the requirements in terms of bandwidth, availability, and latency of the new network management solution. However, further studies should be stimulated to provide an answer to the above-mentioned question.
The paper [16] also outlined two other important elements to be taken into account when considering the application of LEO constellations for the power distribution network: 1) even if the trend for the "power utility Intranet" will be, almost certainly, independent of the public Internet, it will be most likely IP-based and 2) any satellite-based solution must properly interface with legacy wired/wireless systems to provide backward compatibility and transparent communication service. The use of IP over satellite and the interoperability with terrestrial systems will be further discussed in the next sections.

Finally, it is worth mentioning that several smart grid applications foresee command delivery to a group of actuators: this represents a natural application scenario for group-based communications. This way, signaling congestion could be reduced and communication loads optimized if those nodes could be logically grouped based on their service requirements or physical location. This possibility will be further discussed in Section V.

\section{B. Environmental Monitoring}

Health of people and wildlife has been threatened heavily in the last decades. Environmental monitoring aims to enhance the quality of the environment. The use of satellite could be important for the outdoor environmental monitoring in open nature. Outdoor monitoring refers to the detection of the following destructive phenomena: landslides, avalanches, forest fires, volcanoes eruptions, floods, and earthquakes. Such events require fast detection and lead to the need of rapid intervention by first responders (FRs) as discussed in the next section. Furthermore, continuous measurements of air and water pollution, and wildlife position and activity are also included.

Wireless sensor networks (WSNs) are well suited for this class of IoRT applications, such as long-term environmental monitoring [28]. However, environmental monitoring poses stringent requirements on the choice of the WSN: large number of nodes, very low cost, ease of deployment, low maintenance, and very long battery duration (possibly using solar energy). Nodes could be highly mobile (i.e., monitoring of wild animals). On the other hand, requirements on communication delay are quite relaxed since this application does not require real-time operation. In this framework, satellites may play a key role, as they would allow to cover a wide area, where the satellite terminal could be also highly mobile, without installing a complex infrastructure. As a matter of fact, current M2M satellite systems in L band allows mobile applications such as the wildlife monitoring, where sensors are attached to animals that may move over a very large area. While this low data rate and latency-relaxed application scenario seems pretty "easy" for a satellite-based solution, other challenges arise. For instance, in most of the applications, it is of utmost importance to use very energy efficient protocols, in particular MAC protocols. Moreover, MAC algorithms must take into account a very variable topology and number of sensor nodes and that number of nodes might be very high. Finally, the operating cost of the monitoring system should be kept low. Therefore, while current M2M satellite systems may support most of the applications, a more effective use of the satellite resources and a proper access 
to it (via specific multiple access schemes) is needed. Multiple access issues and satellite resources allocation important for this scenario application are discussed in Sections III and IV.

\section{Emergency Management}

Disasters such as earthquakes, fires, floods, explosions, and terrorist attacks may lead to crisis situations which require the intervention of FRs. In order to assist FRs to effectively manage the crisis, emergency response information systems (ERISs) are developed with the aim to provide enhanced situational awareness, automated decision making, and prompt response [29].

Even if the use of existing network infrastructures (PSTN, $2 \mathrm{G}, 3 \mathrm{G}$, and $4 \mathrm{G}$ ) is important to connect a disaster site with the external network (crisis center, data bases with emergency plans, hospitals, etc.), the communication infrastructure at the incident area is often only partially available or completely destroyed. In this context, ensuring radio communications for efficient organization of the relief operations is of paramount importance. A possible solution for this lack of communication means is the deployment of ad hoc wireless networks as an incident area network (IAN), i.e., a self-forming temporary network infrastructure brought to the scene of an incident to support personal and local communications among different public safety end-users (fire brigades, police, medics, etc.) and their connection with a gateway [30]. In this context, the IAN can replace the damaged local terrestrial network infrastructure, and, when connected to a satellite network can provide communication with a remote crisis center. This can guarantee the continuity of standard communications (e.g., voice traffic with the crisis center) and allow the exchange of data related to the particular situation, such as data on location of FRs, alert messages, and electronic maps to support FRs during their motion within the disaster area.

An IAN supports both voice and data transmissions and wireless sensor and actuator communications in the local and/or personal area. The approach to the definition of an IAN for emergency management is the following. Every FR is surrounded by wearable and environmental sensors (e.g., vital sign sensor, temperature sensor, structural health sensor, and indoor/outdoor positioning devices) and actuators (alarms and monitors). The FR is provided with a relief member unit (RMU) which is a network node with multistandard capabilities and gateway functionalities. The wireless standards used by the RMU include 6LoWPAN and TETRA. 6LoWPAN allows the communication of the RMU with sensors and actuators, whereas the TETRA network is used for voice/data communications between RMUs within the IAN.

The end-to-end architecture of ERISs also includes a longrange network (LRN), utilized for the communications between the IAN and the external areas through a transportable gateway. A disaster-proof LRN independent from local terrestrial infrastructure (possibly damaged, destroyed, insufficient, or overloaded) is necessarily based on satellite communication systems (GEO and LEO) [31].

In this scenario, satellite communication systems allow to connect the IAN to Internet, so that emergency services managed by the Emergency Control Center (ECC) can be provided. In particular, the disaster area and the FRs can be remotely monitored by the ECC and several autonomous monitoring and control functions can be provided through sensors and actuators without the human intervention. As for some smart grid scenarios, also in this case, the forward link communication is used for group-oriented services to support both machine- and human-type communications, where concepts such as group-based channel dependent scheduling could be efficiently applied.

Table III summarizes for each of the considered IoRT applications, what can be supported by the current generation of M2M via satellite systems and what are the key issues to be faced to overcome their limits.

\section{Enabling Interconnection Between Satellite AND SENSORS/ACTUATORS}

One of the main enabling factors in satellite communications to support IoRT is the interoperability between satellite and in situ sensors/actuators. As shown in Fig. 2, the satellite collects data measurements from a huge number of sensor nodes and sends it to a ground station for data management. Furthermore, control data are sent from ground stations to the satellite and then to the actuator nodes. Note that, in Fig. 2, we only focus on user data flow without considering signaling/ACK data flow. There are two modes of interoperability between satellite and sensors/actuators: 1) direct access and 2) indirect access. The direct access mode allows sensors and actuators to directly communicate with the satellite, in uplink with the sensors and in downlink with the actuators [32]. In the indirect access mode, each sensor and actuator in a wireless sensor and actuator network (WSAN) may communicate with the satellite through a sink node; therefore, the data flow between the satellite and the WSAN is bidirectional [33]. The advantage of exploiting the indirect access is that a lower number of expensive satellite terminals is required for the same number of sensors/actuators. Furthermore, in the indirect access mode, the sink is provided with a satellite terminal (expensive and power hungry) and with a WSAN radio interface, while all the other nodes of WSAN are only provided with a WSAN radio interface. This solution allows to decrease the system costs and the complexity of the installation (in terms of antenna pointing and power generation facilities). However, this approach has the typical drawbacks of a centralized solution with respect to a decentralized solution.

In current M2M via satellite systems, most of the protocols are proprietary and/or are not designed to support Internet protocols. However, they could also be based on the DVB-S2 standard for the forward link (i.e., the link from the ground station to the actuators) and on the DVB-RCS2 standard for the return link (i.e., the link from the sensors to the ground station) [13], [34]. A novel option is represented by 3GPP long-term evolution (LTE) over satellite [35].

IEEE and IETF standardization activities regarding IoT focused on PHY/MAC layers for low rate wireless personal area networks (IEEE802.15.4 LR-WPAN), convergence layers for IPv6 over low-power WPAN (6LoWPAN), and routing over low-power and lossy networks (ROLLs) [36], [37]. Up to now, 
TABLE III

CRITICAl ISSUES FOR EACH APPLICATION SCENARIO

\begin{tabular}{|c|c|c|c|}
\hline $\begin{array}{l}\text { Application } \\
\text { area }\end{array}$ & $\begin{array}{l}\text { Specific } \\
\text { application }\end{array}$ & $\begin{array}{l}\text { Applicability } \\
\text { of current } \\
\text { M2M services } \\
\text { via satellite }\end{array}$ & Critical issues \\
\hline \multirow[t]{3}{*}{ Smart grid } & $\begin{array}{l}\text { Structural } \\
\text { and } \\
\text { functional } \\
\text { monitoring } \\
\text { of offshore } \\
\text { windfarms }\end{array}$ & $\begin{array}{l}\text { It can be } \\
\text { supported }\end{array}$ & None \\
\hline & $\begin{array}{l}\text { Redundant } \\
\text { path in case } \\
\text { of very high } \\
\text { reliable } \\
\text { links }\end{array}$ & $\begin{array}{l}\text { It can be } \\
\text { supported }\end{array}$ & None \\
\hline & $\begin{array}{l}\text { Remote } \\
\text { monitoring } \\
\text { and } \\
\text { automated } \\
\text { control of } \\
\text { remote } \\
\text { substations }\end{array}$ & $\begin{array}{l}\text { Latency and } \\
\text { bandwidth } \\
\text { requirement } \\
\text { s are not } \\
\text { met }\end{array}$ & $\begin{array}{l}\text { Latency of } \\
\text { TCP/IP-based } \\
\text { satellite networks } \\
\text { Interoperability } \\
\text { with legacy and } \\
\text { terrestrial systems } \\
\text { Group-based } \\
\text { channel-aware } \\
\text { scheduling, radio } \\
\text { resource } \\
\text { management, and } \\
\text { transmission } \\
\text { signaling }\end{array}$ \\
\hline $\begin{array}{l}\text { Environmenta } \\
1 \text { monitoring }\end{array}$ & $\begin{array}{l}\text { Event- } \\
\text { driven, } \\
\text { loose, and } \\
\text { frequent } \\
\text { periodic } \\
\text { reporting }\end{array}$ & $\begin{array}{l}\text { It is } \\
\text { supported }\end{array}$ & $\begin{array}{l}\text { More efficient } \\
\text { solutions in terms } \\
\text { of access to } \\
\text { satellite must be } \\
\text { found to meet the } \\
\text { energy efficiency } \\
\text { challenge, the } \\
\text { varying topology, } \\
\text { and high number of } \\
\text { nodes in several } \\
\text { applications. }\end{array}$ \\
\hline \multirow[t]{2}{*}{$\begin{array}{l}\text { Emergency } \\
\text { management }\end{array}$} & $\begin{array}{l}\text { Structural } \\
\text { monitoring } \\
\text { of the } \\
\text { incident } \\
\text { area } \\
\end{array}$ & $\begin{array}{l}\text { It can be } \\
\text { supported }\end{array}$ & None \\
\hline & $\begin{array}{l}\text { Transmissio } \\
\mathrm{n} \text { of control } \\
\text { messages } \\
\text { from ECC } \\
\text { to actuators } \\
\text { in the } \\
\text { incident } \\
\text { area }\end{array}$ & $\begin{array}{l}\text { Latency } \\
\text { requirement } \\
\text { s are not } \\
\text { met }\end{array}$ & $\begin{array}{l}\text { Latency of } \\
\text { TCP/IP-based } \\
\text { satellite networks } \\
\text { Interoperability } \\
\text { with terrestrial } \\
\text { networks is crucial } \\
\text { Group-based } \\
\text { channel depending } \\
\text { scheduling and } \\
\text { latency-oriented } \\
\text { signaling } \\
\text { mechanisms } \\
\text { M2M traffic } \\
\text { interaction and } \\
\text { balancing }\end{array}$ \\
\hline
\end{tabular}

the IoT standards did not consider any satellite segment in the overall architecture and, therefore, we are dealing with an open research area.

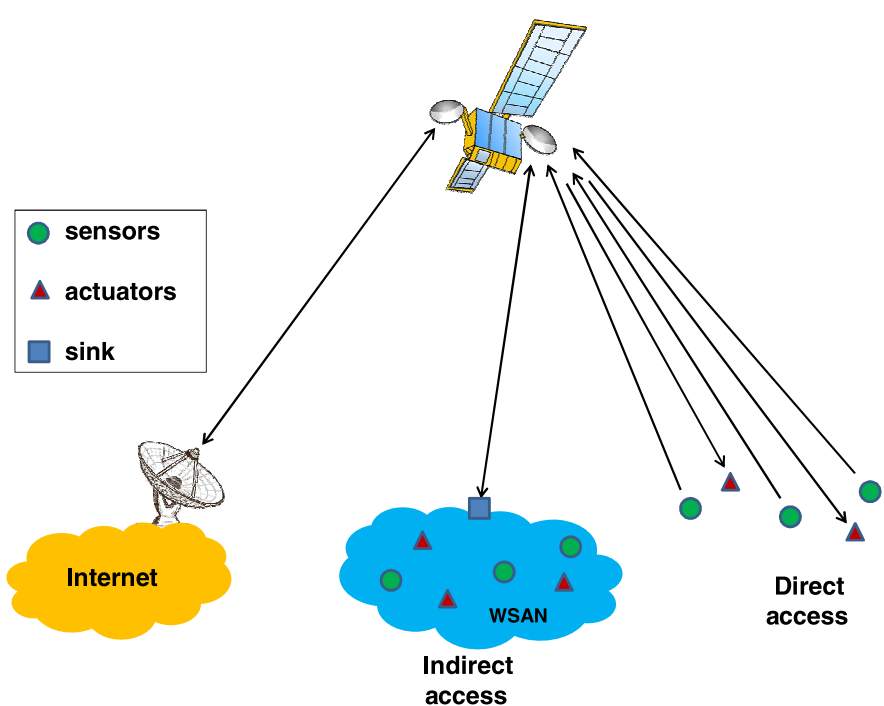

Fig. 2. Example of a satellite system communicating with sensors and actuators.

Two topics are worth considering in this framework: 1) the development of specialized MAC protocols for the access of sensor nodes to satellite resources and 2) the support of IPv6 over satellite. These topics are discussed below.

\section{A. MAC Protocols for Satellite-Routed Sensor and Actuator Networks}

Since sensor nodes are attached to various measuring objects, the data traffic is also various, ranging from periodic to eventdriven data generation. The specific data traffic and data flow direction also depend on the type of access (direct or indirect as in Fig. 2) and the type of application scenario. Choosing the multiple access scheme that can efficiently use satellite resources according to the data traffic and type of access is an important issue. In general, when energy efficiency is very important, TDMA is the well-suited multiple access scheme. On the other hand, in many IoT scenarios, where there is a very high numbers of nodes, but not all of them have data to send, a fixed-assignment multiple access scheme such as TDMA is not efficient.

DVB-RCS2 allows both contention-free and contentionbased multiple access. A set of multifrequency time division multiple access (MF-TDMA) satellite channels is used for communication toward the ground station (gateway). MF-TDMA channels are coordinated using demand assigned multiple access (DAMA) to share the satellite capacity between the set of active user terminals (sensor nodes). The network control center (NCC) controls when each sensor node may transmit. Only one sensor node can access one timeslot in the return channel. If allocation is made to a sensor node that has no data to transmit, then the capacity is wasted, conversely a sensor node with data to transmit, but no allocated timeslots have to wait until the next allocation is received.

Two methods are considered for random access in the DVB-RCS2 standard: 1) slotted ALOHA and 2) contention resolution diversity slotted ALOHA (CRDSA). In CRDSA, 
timeslots are grouped to form a frame [38]. A terminal generates set of replicas of a packet, which are sent using a pseudo-random timeslot within a frame. Several enhancements of the CRDSA have been proposed and investigated [39], [40]. An in-depth analysis of a return link multiple access scheme able to achieve the objectives of low terminal cost and high transmission efficiencies for M2M communications is presented in [41]. The proposed return link multiple access scheme employs asynchronous spread spectrum ALOHA (SSA) techniques combined with low-rate forward error correction (FEC) and downlink channel quality-based transmit ON-OFF packet control at the user side.

In [32], a new algorithm is proposed for a satellite to collect data efficiently from sensor nodes through a direct access. In their proposal, the satellite allocates time slots on demand to sensor nodes which have data to send using a divide and conquer method consisting of a searching phase and an allocation phase. In the searching phase, the satellite finds the sensor nodes having data to send and divide the sensor nodes into two groups. This process is repeated a certain number $D$ of times and each time a group does not include any sensor node which has data to transmit, then this group is removed. The process is repeated no more than $D$ times in order to avoid unnecessary time for confirming the existence of data to send. In the allocation phase, the satellite allocates time slots to all the sensor nodes of the remaining groups regardless of them having data to send or not. It was demonstrated that this algorithm achieves higher efficiency of bandwidth utilization with respect to a TDMA-based fixed assignments scheme or a slotted ALOHA scheme. Indirect access to satellite is considered in [42], where sensor data are collected for a precision agriculture application. In [42], a MAC protocol is proposed which saves the overall energy consumption during the synchronization phase since the protocol uses a relatively short pulse for wake-up signal.

Moreover, it is worth mentioning that there is an increasing interest in using low-cost small satellites $(<50 \mathrm{~kg})$ in LEO, e.g., nanosatellites or CubeSats. Two important advantages provided by nanosatellite constellations are: 1) the possibility to launch them as secondary payloads which greatly reduce the launch cost and 2) they can be built integrating low cost offthe-shelf components even if they are specifically designed for satellite/space applications. When they are launched as secondary payloads, it is not possible to deploy the constellation with the desired orbital characteristics. Moreover, power, mass, and volume constraints pose many challenges in the design of the communication links. Many launched CubeSats use the AX25 standard in the amateur frequency bands with a data rate of $9.6 \mathrm{~kb} / \mathrm{s}$. However, recently also higher (S-band) frequencies have been considered (providing higher data rates). The low data rates, the limited contact of time, and the difficulty to carefully plan the coverage of a given area leads to usually very intermittent and disruptive communication links which makes complex the design of the multiple access. In [43], to address some of these challenges, a communications architecture based on delay tolerant networks (DTNs) protocols has been proposed, which makes use of a new protocol named ALOHA multiple access with gateway priority (ALOHAGP) at the link layer. This solution can be applied to delay-tolerant applications (for instance, environmental monitoring) but not to more time-critical applications such as in smart grid.

\section{B. IPv6 Over Satellite}

The use of IPv6 is the distinctive element of IoT with respect to M2M. IPv6's huge increase in address space is an important factor in the development of the IoT in order to be able to assign an IP address to billions of future simultaneously connected objects.

DVB-RCS2 supports IPv6 and uses generic stream encapsulation (GSE) to reduce the levels of encapsulation and improve efficiency [44]. In DVB-RCS2 standard, IPv6 packets received on the LAN interface of a return channel satellite terminal (RCST) are forwarded according to the RCST IPv6 routing table. Packets may also be redirected to an internal agent (e.g., performance-enhancing Proxy-PEP and intercepting proxy) for processing prior to transmission. Packets for transmission over the air interface are forwarded to the QoS module for transmission over the satellite.

One of the challenges is how to evolve from current IP networking over satellite toward the next-generation network over satellites. One common solution is tunneling. Tunneling IPv6 in IPv4 is a technique used to encapsulate IPv6 packets into IPv4 packets with protocol field 41 of the IP packet header. The tunnel endpoints take care of the encapsulation trough a dual stack and this process is transparent to the intermediate nodes. This solution, however, generates great overhead [15].

It is also worth mentioning that in light of the future Internet (FI) applications domains, where also satellite will play an important role, a novel networking paradigm, alternative to the IP-based internetworking, is emerging: namely the information-centric networking (ICN). ICN is based on accessing named-content in the network, instead of host-to-host communication as in today's Internet. There are several ICN approaches which are currently under investigation in the context of the FI, such as the publish-subscribe Internet routing paradigm (PSIRP), content-centric networking (CCN), named data networking (NDN), network of information (NetInf), dataoriented network architecture (DONA), and cache-and-forward (CNF) [4], [5].

In [45], the applicability of ICN to integrated satellite/terrestrial networks, focusing on the publish-subscribebased ICN approach is investigated. In [45], it is shown that applying the ICN paradigm to a geostationary satellite network can lead to bandwidth savings, greater than those obtained with traditional HTTP means.

\section{INTEROPERABILITy ISSUES BETWEEN SATELLITE AND WiRELESS TERRESTRIAL COMMUNICATION NETWORKS}

\section{A. General Considerations About IoRT Heterogeneity and Related State-of-the-Art}

An obvious characteristic of IoRT is the heterogeneity, i.e., heterogeneous devices communicating through heterogeneous communications networks. Even if the heterogeneity in 
telecommunication networks is an explored research area in terms of architectures and algorithms [46], the IoRT opens new technical issues and research challenges. In more detail, with the inclusion of a wide plethora of new interconnected heterogeneous devices, when QoS is required, the definition of common specific protocols and of the communications modalities between different portions of the IoRT network is necessary. In general, the framework of heterogeneous networks and the QoS concept is developed in [47] and [48], discussing problems and solutions concerning, in particular, resource allocation. Starting from the mentioned literature, each single protocol layer of the IoRT should be involved in this process together with the definition of common interfaces between layers. In the case of satellite networks supporting the IoRT, a suitable solution could be inspired to the so-called ETSI SES BSM for satellites: the layers whose action is strictly dependent of the transmission technology (MAC and PHY layers) are considered divided from the layers whose action does not depend on the specific technology of choice (e.g., case of IP and upper layers) [49]. The proposed distinction implies the so-called problem of the heterogeneous interworking that received specific attention in the literature (see, among the others, [47], [50]-[52], and references therein). All the mentioned works do not directly take into account the possible variations arising from the strong heterogeneity of an IoRT, whose nodes (i.e., the devices) may have severe computational and energy constraints. Indeed, they can heavily affect the overall network performance. For this reason, the following sections are dedicated to a perspective description of solutions (in terms of protocols, QoS and resource management in Section IV-B, and QoS mapping in Section IV-C) aimed at highlighting the specific requirements arisen from their application to the IoRT supported by satellite networks.

\section{B. Satellite/Terrestrial Networks Integration Solutions for IoRT Support}

1) Protocols: Within the mentioned heterogeneous IoRT network, the design of specialized protocols is topical. Novel solutions may be applied to each protocol layer. To achieve satellite-based IoRT QoS solution, each single protocol should be optimized. In this context, PHY and MAC layers play a crucial role concerning the transmission resource (e.g., bandwidth) management. The network layer has to efficiently use the bandwidth offered by the lower layers and implement QoS reservation and QoS mapping mechanisms, while the transport and application protocols must efficiently use the resources offered by the network layer. In this view, a cross-layer approach represents a required solution to be applied in such a heterogeneous IoRT. In practice, the cross-layer approach allows protocol entity to exploit the knowledge of a set of parameters (measured or estimated) from the underlying layers, providing an optimization framework involving all the layers from the physical up to the transport.

2) QoS Mapping: In a heterogeneous satellite-based IoRT, QoS mapping is usually needed "vertically" (i.e., the MAC layer should offer a service to the network layer) and "horizontally" (i.e., connecting the radio and satellite technologies and QoS mechanisms through a proper signaling scheme). In

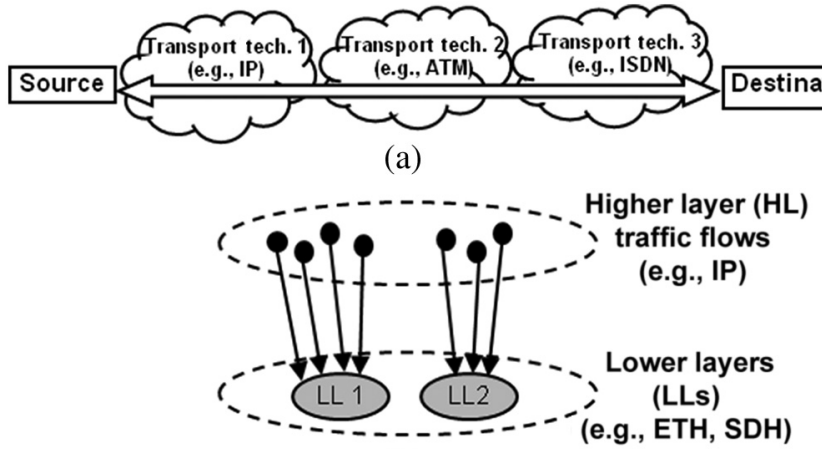

(b)

Fig. 3. Horizontal and vertical QoS mapping concepts. (a) Horizontal QoS mapping. (b) Vertical QoS mapping.

Fig. 3, the general concept of the two QoS mappings is reported. Specific details about the related concepts and techniques can be found in [46].

In the case of vertical QoS mapping, an interface (network/datalink) has to be defined, to manage the mapping of QoS specification imposed at the network layer (e.g., DiffServ architecture) to the class of service $(\mathrm{CoS})$ structure that should be defined within the datalink layer. As far as the horizontal QoS mapping is concerned, solutions take into account the technological heterogeneity of the possible radio access network (RAN) of an IoRT. Indeed, in a satellite-based IoRT, a node (or device) might employ the DVB-S2/DVB-RCS2 technology to access the satellite portion of the network while TETRA, WiMAX, Wi-Fi, or other wireless technologies to access the terrestrial portions of the IoRT.

3) Resource Allocation: The above-mentioned need to guarantee a certain QoS has driven the development of dynamic bandwidth allocation techniques, which take into account the current status of the channel employed to access the IoRT. In more detail, satellite environments linked to the mentioned specific technologies are characterized by several peculiarities, which imply the introduction of suitable control strategies. Satellite channels vary their characteristics depending on the weather, and the effect of fading heavily affects the performance of the whole system. The practical effect is on the QoS offered to the users exploiting the IoRT devices. To avoid this condition, techniques aimed at providing compensation for rain attenuation are needed [12]. Bandwidth allocation is strictly related to the MAC scheme and must be defined by finding a compromise between QoS and other metrics characterizing the "things" of the IoRT. Indeed, battery lifetime in the case of mobile devices, or fair employment of the network resources, are fundamental issues to be taken into account [53], [54].

\section{QoS-Oriented Solutions: TI-SAP}

Protocol stacks of the devices in the above-mentioned scenarios are composed of a great heterogeneity of functional layers. For this reason, a common language to support QoS along the end to end path is crucial. Such a notion of "common language" is also known in the literature as the aforementioned QoS mapping. 
Concerning the vertical QoS mapping, an applicable solution is the so-called technological independent-service access point (TI-SAP) that can be used as a reliable solution for the IoRT [46]. The main concept of the TI-SAP is the queue identifiers (QIDs) which represents an abstract queue at the TI-SAP level. Each QID is formally a relationship between IP queues (i.e., DiffServ queues) and technology-dependent (TD) queues (i.e., MAC queues). Each QID defines a CoS characterizing IP packets to be transferred into the TD core. The application of QID principle allows hiding to TI layers the local implementation of the QoS within the TD systems, where, for instance, DVB (for the satellite portions) or Wi-Fi (for some terrestrial portions) may be applied. A QID represents how a specific subset of IP queues are mapped over a specific subset of TD queues. The definition of the TI-SAP functionalities through proper QID primitives to support resource allocation and control has the main role to solve the above-mentioned vertical QoS mapping problem. It is also worth noting that specific operations shall be performed at the control plane level of the TI-SAP protocol dealing also with the horizontal QoS mapping. More specifically, a proper signaling mechanism is needed to support both QoS mappings. Possible examples may be resource reservation protocol - traffic engineering (RSVP-TE) - if the TI-SAP interfaces multiprotocol label switching (MPLS) networks, or extensions made on RSVP to support call admission control (CAC) information in DiffServ environments (called RSVPpre congestion notification) [55]-[57]. In this perspective, novel control methodologies need to be proposed to support bandwidth adaptation when QoS mapping operations are applied in a heterogeneous IoRT.

\section{Group-Based Delivery in M2M SATEllite ENVIRONMENT}

As mentioned in Section II, several applications for M2M will exploit the forward link for delivering commands and control information via satellite to the huge number of actuators/smart objects deployed in the environment [58]. Imagine a smart grid scenario where the great number of actuators could be suitably remotely controlled for efficiently managing the power production, transmission, and distribution. In emergency management systems, the forward link communication could be used for providing group-oriented services to support both MTC (i.e., alert commands to the actuators deployed to a large, rural and inaccessible areas, or the simultaneous delivery of software updates such as firmware toward a large of machines) and human-type communications (i.e., for updating periodically FRs about their position in disaster area, for providing electronic map to support FRs during their motion and/or for forecast/security news delivery). These represent only a subset of the possible applications that in the downlink direction could efficiently exploit the large coverage provided by satellite systems.

In the depicted scenarios, the primary goal of group-based communications is to alleviate the signaling overhead on both radio access and core network segments. Indeed, actuators and smart devices that need to receive the same downlink control messages (e.g., commands for actuators) and/or data packets (e.g., firmware/configuration or information file download) can be logically grouped based on their service requirements (i.e., multicasting scenarios) or their physical location (i.e., geocasting scenarios), to reduce the signaling congestion on the air interface and to optimize the communication loads [59]. Moreover, a further important effect is to reduce the overall power consumption for users, machines, and base stations.

Therefore, the definition of efficient radio resource management strategies is challenging, because the same spectrum is expected to be utilized for human-to-human $(\mathrm{H} 2 \mathrm{H})$ communications as well as M2M communications [60].

Furthermore, the effective provisioning of group-based M2M communication via satellite involves several challenges in terms of traffic scheduling, mainly due to different channel conditions experienced by the $\mathrm{M} 2 \mathrm{M} / \mathrm{H} 2 \mathrm{H}$ group within the same spotbeam [60]. Generally, the nodes with good channel conditions are able to support transmission with high spectral efficiency (i.e., high data rate for a given bandwidth), whereas the ones undergoing a bad channel must be served using a low spectral efficiency transmission (i.e., low data rate for a given bandwidth) since they must be more robust to channel errors.

Concerning the conservative approach [60], M2M devices involved in the group communication are served according to the node with the poorest channel quality. Although this approach could maximize the fairness among M2M devices and is robust to channel quality variations, it introduces severe performance degradation in terms of spectrum efficiency.

In alternative, the opportunistic approach [60] foresees to serve, in any given time slot, only a portion of nodes to maximize a given cost function, such as system throughput. The goal of this approach is to exploit the multinode diversity in resource allocation, although it may limit the number of M2M devices successfully served in each time slot. As a consequence, to avoid a severe limitation of the involved nodes, additional efficient and robust data coding (e.g., rateless codes) can be employed to this scope. Although opportunistic approaches can achieve long-term fairness (which can be considered suitable in delay tolerant applications, such as file delivery), it cannot achieve short-term fairness (since not all nodes are served within every time slot) that, on contrary, is more important in delay sensitive applications [60].

Another interesting approach that overcomes the limitations of conservative and opportunistic schemes is the subgrouping [59], which foresees to serve all M2M devices belonging to group communication in every time slot and to split the nodes into different subgroups according to the experienced channel conditions. The subgroup formation is performed to take advantage of the multinode diversity by guaranteeing improved session quality for the users with good channel qualities.

\section{CONCLUSION}

This paper provides an overview of the research activity in satellite communication in view of its use in IoRT application scenarios. Important issues that have been discussed are: heterogeneous networks interoperability, QoS management, group-based communications, and efficient IPv6 support. For each of these topics, results of some recent studies have been reported and some further directions of research have been identified. 
As a matter of fact, there is no much research specifically focused on the application of satellite to IoRT scenarios and the authors feel that there is no real awareness of the potential advantages coming from their use in the future IoRT scenarios, even if they already play an important role in several M2M communication applications (energy sector, oil and gas, and logistics) nowadays. Only few feasibility studies have shown their possible use in future IoRT application scenario (for instance, for the smart grid [16]). The authors believe that some more effort should be spent in this direction. For instance, as already mentioned, there is a growing interest in understanding the potential use of constellations of nanosatellites. It could be worth trying to understand if those constellations of nanosatellites could be a real cost-benefit solution in some specific IoRT scenario.

\section{REFERENCES}

[1] J. A. Stankovic, "Research directions for the Internet of Things," IEEE Internet Things J., vol. 1, no. 1, pp. 3-9, Feb. 2014.

[2] M. De Sanctis, C. Stallo, S. Parracino, M. Ruggieri, and R. Prasad, "Interoperability solutions between smartphones and wireless sensor networks," in Proc. IEEE 1st AESS Eur. Conf. Satell. Telecommun. (ESTEL'12), Rome, Italy, Oct. 2-5, 2012, pp. 1-6.

[3] S. Y. Lien, K. C. Chen, and Y. Lin, "Toward ubiquitous massive accesses in 3GPP machine-to-machine communications," IEEE Commun. Mag., vol. 49, no. 4, pp. 66-74, Apr. 2011.

[4] G. Fairhurst, L. Caviglione, and B. Collini-Nocker, "FIRST: Future Internet-A role for satellite technology," IEEE Int. Workshop Satell. Space Commun. (IWSSC'08), Oct. 1-3, 2008, pp. 160-164.

[5] K. Liolis, N. Chuberre, I. Andrikopoulos, and M. Piccinni, "On a future Internet architecture augmented by satellite networks," in Proc. Future Netw. Mobile Summit, Jun. 16-18, 2010, pp. 1-8.

[6] F. Ghavimi and C. Hsiao-Hwa, "M2M communications in 3GPP LTE/LTE-A networks: Architectures, service requirements, challenges and applications," IEEE Commun. Surveys Tuts., vol. 17, no. 2, pp. 525549, May 2015.

[7] NetWorld2020's whitepaper. (2014, Sep.). "5G: Challenges, research priorities, and recommendations," [Online]. Available: http://networld2020. eu/sria-and-whitepapers/

[8] "Satellite machine-to-machine (M2M) services-Global strategic business report," Global Ind. Anal. Inc., San Jose, CA, USA, Mar. 2012, $484 \mathrm{pp}$.

[9] ORBCOMM. (2015). "M2M service delivery simplified," [Online]. Available: http://www.orbcomm.com

[10] B. R. Elbert, The Satellite Communication Applications Handbook. Norwood, MA, USA: Artech House, 2004, ch. 9.

[11] S. D. Ilcev, Global Mobile Satellite Communications: For Maritime, Land and Aeronautical Applications. New York, NY, USA: Springer, 2005, ch. 6.

[12] S. Mukherjee, M. De Sanctis, T. Rossi, E. Cianca, M. Ruggieri, and R. Prasad, "Mode switching algorithms for DVB-S2 links in W band," in Proc. IEEE Global Telecommun. Conf. (Globecom'10), Miami, FL, USA, Dec. 6-10, 2010.

[13] ETSI EN 302-307 vl.1.1, "Digital video broadcasting (DVB); second generation framing structure, channel coding and modulation systems for broadcasting, interactive services, news gathering and other broadband satellite applications," 2004.

[14] H. Zhou, The Internet of Things in the Cloud: A Middleware Perspective. Boca Raton, FL, USA: CRC, 2012, ch. 4.

[15] D. Minoli, Building the Internet of Things With IPv6 and MIPv6: The Evolving World of M2M Communications. Hoboken, NJ, USA: Wiley, 2013, ch. 4.

[16] Q. Yang, D. I. Laurenson, and J. A. Barria, "On the use of LEO satellite constellation for active network management in power distribution networks," IEEE Trans. Smart Grid, vol. 3, no. 3, pp. 1371-1381, Sep. 2012.

[17] G. Cocco and C. Ibars, "On the feasibility of satellite M2M systems," in Proc. 30th AIAA Int. Commun. Satell. Syst. Conf. (ICSSC'12), Ottawa, ON, Canada, Sep. 24-27, 2012, pp. 1-11.

[18] O. Vermesan and P. Friess, Eds., Internet of Things-Global Technological and Societal Trends. Aalborg, Denmark: River, 2011.
[19] "European technology platform SmartGrids: Vision and strategy for Europe's electricity networks of the future," Eur. Commission Rep., Directorate-General for Res. Sustainable Energy Syst., 2006.

[20] B. Fairbanks, "SCADA application for ACTS technology," in Proc. Nat. Telesyst. Conf. (NTC'92), May 19-20, 1992, pp. 8/15-8/21.

[21] J. Marihart, "Communications technology guidelines for EMS/SCADA systems," IEEE Trans. Power Del., vol. 16, no. 2, pp. 181-188, Apr. 2001.

[22] P. V. F. Beardow, J. A. Barber, R. Owen, and J. C. Bell, "The application of satellite communications technology to the protection of the rural distribution networks," in Proc. 5th Int. Conf. Develop. Power Syst. Protection, Mar. 30-Apr. 1, 1993, pp. 17-20.

[23] R. Duke-Woolley, "Findings from ESA SAMOS project—Satellite M2m observatory study," in Proc. 26th Adv. Satell. Multimedia Syst. Conf. $($ ASMS)/12th Signal Process. Space Commun. Workshop (SPSC), Sep. 5-7, 2012, pp. 13-20.

[24] R. J. F. Fang, M. Eroz, and N. Becker, "Data collection and SCADA over GEO-MSS satellites using spread SCMA," in Proc. Int. Workshop Satell. Space Commun. (IWSSC'09), Sep. 9-11, 2009, pp. 441-445.

[25] X. Xiao, Z. Fu, G. Liu, and C. Deng, "A backup data network for power system automations based on satellite communication," in Proc. Int. Conf. Power Syst. Technol. (POWERCON), Oct. 24-28, 2010. pp. 1-5.

[26] M. Chenine and L. Nordstrom, "Modeling and simulation of wide-area communication for centralized PMU-based applications," IEEE Trans. Power Del., vol. 26, no. 3, pp. 1372-1380, Jul. 2011.

[27] A. Vaccaro and D. Villacci, "Performance analysis of low earth orbit satellites for power system communications," Elect. Power Syst. Res., vol. 73, no. 3, pp. 287-294, 2005.

[28] M. Lazarescu, "Design of a WSN platform for long-term environmental monitoring for IoT applications," IEEE J. Emerging Sel. Topics Circuits Syst., vol. 3, no. 1, pp. 44-54, Mar. 2013.

[29] L. Yang, S. H. Yang, and L. Plotnick, "How the Internet of Things technology enhances emergency response operations," Technol. Forecasting Social Change Int. J., vol. 80, no. 9, pp. 1854-1867, Nov. 2013.

[30] G. Araniti et al., "Cooperative terminals for incident area networks," in Proc. 1st Int. Conf. Wireless (VITAE'09), Aalborg, Denmark, May 17-20, 2009, pp. 549-553.

[31] G. Araniti et al., "Hybrid system HAP-WiFi for incident area network," in Proc. 2nd Int. Conf. Pers. Satell. Serv. (PSATS'10), Rome, Italy, Feb. 4-5, 2010, pp. 436-450.

[32] Y. Kawamoto, H. Nishiyama, Z. M. Fadlullah, and N. Kato, "Effective data collection via satellite-routed sensor system (SRSS) to realize globalscaled Internet of Things," IEEE Sensors J., vol. 13, no. 10, pp. 36453654, Oct. 2013.

[33] I. Bisio and M. Marchese, "Efficient satellite-based sensor networks for information retrieval," IEEE Syst. J., vol. 2, no. 4, pp. 464-475, Dec. 2008.

[34] ETSI EN 301-545-2, "Digital video broadcasting (DVB); second generation DVB interactive satellite system (DVB-RCS2); part 2: lower layers for satellite standard," 2012.

[35] M. Papaleo, M. Neri, A. Vanelli-Coralli, and G. Corazza, "Using LTE in 4G satellite communications: Increasing time diversity through forced retransmission," in Proc. 10th Int. Workshop Signal Process. Space Commun. (SPSC'08), Oct. 6-8, 2008, pp. 1-4.

[36] M. R. Palattella et al., "Standardized protocol stack for the Internet of (important) Things," IEEE Commun. Surveys Tuts., vol. 15, no. 3, pp. 1389-1406, Dec. 12, 2012.

[37] I. Ishaq et al., "IETF standardization in the field of the Internet of Things (IoT): A survey," J. Sensor Actuator Netw., vol. 2, no. 2, pp. 235-287, Apr. 25, 2013.

[38] E. Casini, R. De Gaudenzi, and O. R. Herrero, "Contention resolution diversity slotted ALOHA (CRDSA): An enhanced random access scheme for satellite access packet networks," IEEE Trans. Wireless Commun., vol. 6, no. 4, pp. 1408-1419, Apr. 2007.

[39] O. del Rio Herrero and R. De Gaudenzi, "High performance MAC protocol for consumer broadband satellite systems," in Proc. 27th AIAA Int. Commun. Satell. Syst. Conf., Edinburgh, U.K., Jun. 2009, pp. 512-524.

[40] G. Liva, "Graph-based analysis and optimization of contention resolution diversity slotted ALOHA," IEEE Trans. Commun., vol. 59, no. 2, pp. $477-$ 487, Feb. 2011.

[41] O. del Rio Herrero and R. De Gaudenzi, "High efficiency satellite multiple access scheme for machine-to-machine communications," IEEE Trans. AESS, vol. 48, no. 4, pp. 2961-2989, Oct. 2012.

[42] H. Sahota, R. Kumar, and A. Kamal, "Performance modeling and simulation studies of MAC protocols in sensor network performance," in Proc. 7th Int. Wireless Commun. Mobile Comput. Conf. (IWCMC), Jul. 4-8, 2011, pp. 1871-1876. 
[43] H. Bedon, C. Miguel, A. Fernandez, and J. S. Park, "A DTN system for nanosatellite-based sensor networks using a new ALOHA multiple access with gateway priority," Smart Comput. Rev., vol. 3, no. 5, pp. 383-396, Oct. 2013.

[44] ETSI TS 101 545-3, "Digital video broadcasting (DVB); second generation DVB interactive satellite system (DVB-RCS2); part 3: higher layers satellite specification," 2012.

[45] A. Detti, A. Caponi, and N. Blefari Melazzi, "Exploitation of information centric networking principles in satellite networks," in Proc. IEEE 1st AESS Eur. Conf. Satell. Telecommun. (ESTEL), Oct. 2-5, 2012, pp. 1-6.

[46] M. Marchese, QoS Over Heterogeneous Networks. Hoboken, NJ, USA: Wiley, Jun. 2007

[47] M. P. Howarth et al., "End-to-end quality of service provisioning through inter-provider traffic engineering," Comput. Commun., vol. 29, no. 6 , pp. 683-702, Mar. 2006.

[48] Tacoms Post 2000Project (TACOMS), TACOMS STANAG 4637. (2005). TACOMS HEAD STANAG (draft ed. 1), NATO Steering Committee, NSA [Online]. Available: http://www.tacomspost2000.org

[49] ETSI TS 102-462 v0.4.2, "Satellite earth stations and systems. broadband satellite multimedia services and architectures: QoS functional architecture," Tech. Specification, Jan. 2006.

[50] J. Schmitt, "Translation of specification units between IP and ATM quality of service declarations," Int. J. Commun. Syst., vol. 16, no. 4 pp. 291-310, 2003.

[51] M. Marchese and M. Mongelli, "On-line bandwidth control for quality of service mapping over satellite independent service access points," Comput. Netw., vol. 50, no. 12, pp. 1885-2126, Aug. 2006.

[52] S. Georgoulas, P. Trimintzios, G. Pavlou, and K. Ho, "Heterogeneous real-time traffic admission control in differentiated services domains," in Proc. IEEE Global Telecommun. Conf. (Globecom'05), St. Louis, MO, USA, Nov. 28-Dec. 2, 2005, pp. 523-528.

[53] I. Bisio, M. Marchese, and G. Portomauro, "Performance evaluation of sink selection techniques in satellite sensor networks," in Proc. IEEE Global Commun. Conf. (GLOBECOM'06), San Francisco, CA, USA, Nov. 27-Dec. 1, 2006, pp. 1-5.

[54] I. Bisio and M. Marchese, "Power saving bandwidth allocation over GEO satellite networks," IEEE Commun. Lett., vol. 16, no. 5, pp. 596-599, May 2012.

[55] E. Rosen, A. Viswanathan, and R. Callon, "Multiprotocol label switching architecture," IETF RFC3031.

[56] D. Awduche, L. Berger, D. Gan, T. Li, V. Srinivasan, and G. Swallow, "RSVP-TE: Extensions to RSVP for LSP tunnels," IETF RFC3209.

[57] P. Eardley, "Metering and marking behaviour of PCN-nodes," IETF RFC5670.

[58] A. Richard, A. Dadlani, and K. Kim, "Multicast scheduling and resource allocation algorithms for OFDMA-based systems: A survey," IEEE Commun. Surveys Tuts., vol. 15, no. 1, pp. 240-254, Feb. 2013.

[59] G. Araniti, V. Scordamaglia, M. Condoluci, A. Molinaro, and A. Iera, "Efficient frequency domain packet scheduler for point-to-multipoint transmissions in LTE networks," in Proc. IEEE Int. Conf. Commun. (ICC), Jun. 2012, pp. 4405-4409.

[60] M. Condoluci, M. Dohler, G. Araniti, A. Molinaro, and K. Zheng, "Toward 5G densenets: Architectural advances for effective machine-type communications over femtocells," Commun. Mag. IEEE, vol. 53, no. 1 , pp. 134-141, Jan. 2015.

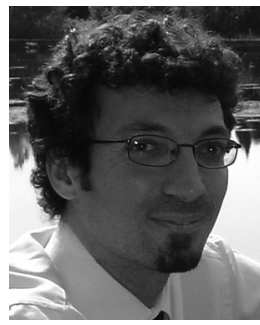

Mauro De Sanctis received the Laurea degree in telecommunications engineering and $\mathrm{Ph} . \mathrm{D}$. degree in telecommunications and microelectronics engineering from the University of Roma "Tor Vergata," Rome, Italy, in 2002 and 2006, respectively.

He has coauthored more than 80 papers published on journals and conference proceedings. His research interest include wireless terrestrial and satellite communication networks, human activity recognition, data mining, and information theory.

Dr. Sanctis is serving as an Associate Editor for the IEEE Aerospace and Electronic Systems Magazine.

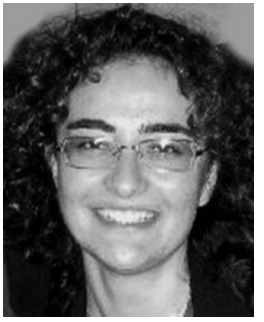

Ernestina Cianca is currently an Assistant Professor with the Department of Electronic Engineering, University of Rome "Tor Vergata," Rome, Italy, and Liaison Office's Chair of the Interdisciplinary Center, Center for TeleInfrastruktur (CTIF), Aalborg, Denmark. Her research interests include wireless access technologies (CDMA, OFDM, SC-FDE) and equalization for both terrestrial and satellite communications, satellite communications in EHF bands (in particular, W-band), and microwave medical imaging. She has a relevant experience in national and European projects. She has authored about 80 papers in international journal and conference proceedings.

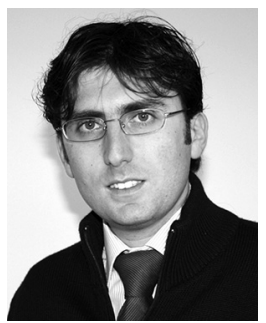

Giuseppe Araniti received the Laurea and Ph.D. degrees in electronic engineering from the University Mediterranea of Reggio Calabria, Reggio Calabria, Italy, in 2000 and 2004, respectively.

$\mathrm{He}$ is currently an Assistant Professor of telecommunications with the University Mediterranea of Reggio Calabria. His research interests include personal communications systems, enhanced wireless and satellite systems, traffic and radio resource management, multicast and broadcast services, deviceto-device, and machine-type communications over $4 \mathrm{G} / 5 \mathrm{G}$ cellular networks.

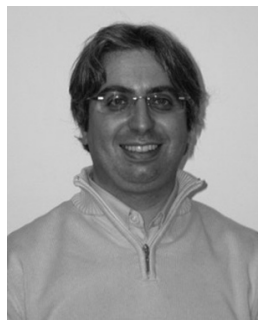

Igor Bisio (S'04-M'07-SM'15) received the Laurea degree in telecommunications engineering and Ph.D. degree in information and communication sciences from the University of Genoa, Genoa, Italy in 2002 and 2006, respectively.

$\mathrm{He}$ is currently an Assistant Professor with the University of Genoa. He has authored or coauthored about 100 scientific papers including international journals, international conferences, and book chapters. His research interests include digital signal processing over smartphone platforms, contextawareness, Internet of Things, and satellite systems.

Dr. Bisio has been the Chair of the IEEE Communications Society Satellite and Space Communications Technical Committee since 2012.

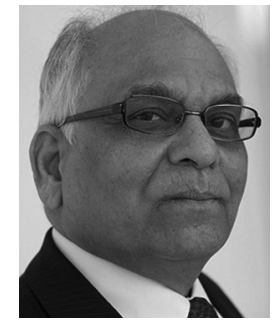

Ramjee Prasad (M'88-SM'90-F'09) is currently the Director of the Center for TeleInfrastruktur (CTIF), Aalborg University, Aalborg, Denmark, and a Professor and Chair of Wireless Information Multimedia Communication.

Prof. Prasad is a Fellow of the Institution of Electronics and Telecommunications Engineers (IETE), India, and the Institution of Engineering and Technology (IET), U.K. He is a Member of the Netherlands Electronics and Radio Society (NERG), and the Danish Engineering Society (IDA). He is the Founding Chairman of the Global ICT Standardisation Forum for India (GISFI: www.gisfi.org) established in 2009. 